\title{
Aging Effect on Korean Female Voice: Acoustic and Perceptual Examinations of Breathiness
}

\author{
Seung Jin Lee ${ }^{a}$ YoonHee Cho $^{a}$ Ji Yeon Song ${ }^{a}$ DamHee Lee ${ }^{b}$ Yunjung Kim ${ }^{d}$ \\ HyangHee Kim ${ }^{\mathrm{a}, \mathrm{c}}$ \\ ${ }^{a}$ Graduate Program in Speech and Language Pathology, Yonsei University, bepartment of Otolaryngology, Asan \\ Medical Center, University of Ulsan College of Medicine, and ${ }^{c}$ Department and Research Institute of Rehabilitation \\ Medicine, Yonsei University College of Medicine, Seoul, Korea; ${ }^{\mathrm{d} D e p a r t m e n t}$ of Communication Sciences and \\ Disorders, Louisiana State University, Baton Rouge, La., USA
}

\section{Key Words}

Voice $\cdot$ Aging $\cdot$ Acoustic measures $\cdot$ Breathiness $\cdot$ Korean females

\section{Abstract \\ Objective: This paper sought to examine perceptual and acoustic characteristics in Korean female voices, focusing on the 'breathy' quality as a function of aging. In addition, we aimed to investigate if the three selected measures, $\mathrm{H} 1-\mathrm{H} 2$, $\mathrm{H} 1-\mathrm{A} 1$, and $\mathrm{H} 1-\mathrm{A} 3$, demonstrated any changes along a sus- tained vowel production. Participants and Methods: A total of 42 participants were assigned to two age groups, young women and elderly women. All participants were asked to sustain /a/ as long and as steadily as possible. Perceptual judgments of breathiness were made on the GRBAS scale and by a direct magnitude estimation technique, while three acoustic parameters, $\mathrm{H} 1-\mathrm{H} 2, \mathrm{H} 1-\mathrm{A} 1$, and $\mathrm{H} 1-\mathrm{A} 3$, were mea- sured at five measurement time points during the sustained vowel test. Results: Results indicated that the $\mathrm{H} 1-\mathrm{H} 2$ and $\mathrm{H} 1-$ A1 values were significantly lower for elderly women com- pared to young women, although no difference in the per- ceptual estimation of breathiness was found between the}

age groups. Among the acoustic measures, only H1-A1 was significantly regressed against the perceptual estimate of breathiness. In addition, no significant acoustic difference in the measures was found across the five measurement points. Conclusion: Our findings suggest that the aging voice might not be universally characterized by the breathy quality, which hints at the need for further research on ethnic diversity in vocal quality.

(c) 2016 S. Karger AG, Basel

\section{Introduction}

The effect of aging on voice change is an increasingly important issue because of the growing population of older people and the increasing concerns about their health and quality of life. Such effects on vocal characteristics have frequently included pitch change, voice tremor, voice breaks, and increased breathiness and/or hoarse-

This work was presented in part at the Asia Pacific Conference of Speech, Language and Hearing 2015, Guangzhou, China, and awarded Best Presentation Award.

\section{KARGER 125}

C 2016 S. Karger AG, Base

$1021-7762 / 16 / 0676-0300 \$ 39.50 / 0$ 
ness $[1,2]$. These perceptual descriptions have also been supported by acoustic findings. For example, pitch change has been identified in elderly speakers, such that women maintain quite constant F0 until menopause, after which F0 decreases approximately by $10-15 \mathrm{~Hz}$, while men exhibit a decrease from young adulthood to middle age, then a substantial increase by approximately $35 \mathrm{~Hz}$ into advanced old age [3, 4]. In addition, reduced stability has been supported by several acoustical measures including jitter, shimmer, fundamental frequency standard deviation, and amplitude perturbation quotient $[5,6]$.

The current study focuses on the vocal quality of breathiness in Korean elderly females for the following reasons. First, despite the general assumption that the elderly exhibit breathy voice, experimental studies (including perceptual and acoustic) have shown considerable disagreement, especially for women $[1,4,7,8]$. These conflicting findings may reflect the fact that the underlying mechanisms of breathy voice in elderly women (EW) are not clearly understood. In general, the presence of incomplete glottal closure and subsequent 'air leakage' is assumed to be associated with the perception of breathiness $[9,10]$. Unlike men in whom the degree of glottal gap seems to differ clearly between the young and elderly, both young women (YW) and EW have frequently been reported to demonstrate a high incidence of glottal gap, even though gap location may differ across age. Previous data have suggested that YW tend to have a posterior chink, while EW have the gap anteriorly $[5,11]$.

Second, we sought to examine this widely accepted vocal quality change due to aging in an underrepresented ethnic group in the literature, in recognition that prior work has been conducted primarily in certain ethnic groups such as European American [7, 12], Brazilian [13, 14], and Indian [15] populations, although several studies have suggested that voice quality varies enough among ethnic groups to serve as an identification marker of ethnicity or native language [16-19]. One possible reason for this is that factors affecting vocal quality (e.g., age at menopause) may differ between ethnic groups. In a cohort study, the mean age at menopause in Korean women was 46.9 years, which is earlier than previously reported Caucasian data [20]. Although, to our knowledge, no perceptual or acoustic investigations have been systematically conducted, a very limited number of studies have indirectly implied conflicting results with respect to the degree of breathiness in Korean elderly females [21]. One study of Korean speakers claimed increased breathiness in EW compared to YW based on the former's greater noise-to-harmonic ratio [22], while another study report- ed no difference in the degree of breathiness based on similar Soft Phonation Index (SPI) values between the two groups. SPI is a parameter provided by the MultiDimensional Voice Program (Computerized Speech Lab) as an index of vocal fold approximation during phonation [23]. The inconsistent findings combined with a lack of perceptual data make it difficult to draw firm conclusions, especially considering the controversial interpretation of the two parameters included in the studies. Previous studies have questioned the degree to which these parameters can be reliably and uniquely correlated with breathiness [24].

The final motivation of the study was a desire to gain a better understanding of different acoustic measurement points in the vowel prolongation task, which is routinely used for voice evaluation. Many acoustic measures derived from prolonged vowels are meant to provide correlates of breathiness, but it is possible that these measures vary during the relatively long interval of a sustained vowel. The temporal 'midpoint' (typically a 1- or 3 -second window centered around it) has been widely used in research and the clinic under the assumption that it is the most stable portion of the voice source [25-27]. Given the nature of this maximum performance task, the vocal characteristics might vary over time. Clinical observations that the voice of dysphonic patients frequently tends to be 'pressed' toward the end of exhalation also support this. However, very few studies have systematically investigated vocal changes throughout prolonged vowels.

For this purpose, we chose five ratio-based points of measurement along the vowel productions. In addition to the temporal midpoint of each vowel, acoustic data were sampled at the midpoints of each half of the vowel duration (that is 25 and $75 \%$ points), as well as points $1.5 \mathrm{~s}$ later and earlier than the sustained vowel onset and offset, respectively [28]. It was hypothesized that significant differences in acoustic characteristics may exist across these measurement points, considering the nature of the maximum phonation task which is supposed to reflect the efficiency of respiratory mechanism during phonation [29]. If the choice of the measurement points was associated with systematically varying acoustic results, as hypothesized, the selection of a measurement point for clinical evaluation would need to be done carefully.

Three acoustic variables were selected in the study: $\mathrm{H} 1-\mathrm{H} 2, \mathrm{H} 1-\mathrm{A} 1$, and H1-A3. Previous studies have discussed these measures as physically associated with incomplete closure of the vocal folds and/or a longer open phase during phonation $[12,15,30,31]$. Since Hanson's 
investigation [31] on the relationship between the amplitude of the first harmonic ( $\mathrm{H1}$ ) and the first formant frequency (A1) in young females, the parameter H1-A1 has been investigated as an acoustic correlate of breathiness in other groups of speakers, including elderly voices [7] and pathological voices $[15,32]$. Based on the spectral tilt across the speech frequency range, the amplitude of the third formant frequency (A3) relative to $\mathrm{H} 1$ has been of interest for similar reasons [33-35]. We were interested in how these acoustic measures are correlated with perceptual judgments of breathiness that is often thought to reflect physical or functional abnormality of vocal fold vibration.

To obtain perceptual estimates of breathiness, the GRBAS scale and a direct magnitude estimation (DME) method were employed in the study. The GRBAS scale was selected because it is widely used in voice research and the clinic [36, 37]. However, due to its potential scale attenuation effect (only a 4-point scale), especially given that our participants were all free from voice pathology, a DME estimate was additionally employed. DME was originally developed for the scaling of sensory attributes such as brightness and loudness, in which people assign numbers in proportion to a standard modulus, or to the assigned magnitude of a previously estimated stimulus $[38,39]$.

In sum, the overarching goal of the present study was to examine perceptual and acoustic differences in breathiness between young and elderly Korean female voices. Specific research questions were posed as follows: (1) Are Korean EW perceived as more breathy compared to YW? (2) Do all or some of the selected acoustic variables reflect different degrees of breathiness between the two groups? (3) Do the selected acoustic variables change as a function of time during prolonged vowels?

\section{Methods}

\section{Participants}

A total of 50 community-dwelling Korean females in Seoul and Anyang were initially recruited in the study. All participants were native speakers of Korean. Among them, 8 women were excluded according to the exclusion criteria, which included a self-reported history of (1) smoking or other neurological/health issues, (2) visiting a voice clinic in the previous 3 months, and (3) any clinically relevant voice complaint at the date of voice recording. The 42 remaining women were divided into two groups based on their chronological age: $21 \mathrm{YW}$ (mean age $=21.38$ years; $\mathrm{SD}=3.06$; range $=20-29$ ) and $21 \mathrm{EW}$ (mean age $=74.67$ years; $\mathrm{SD}=4.81$; range $=66-81)$. Informed consent was obtained from all participants.

\section{Voice Recordings}

Voice recordings were made in a silent room using a dynamic microphone (SHURE, SM58) and a digital recorder (SONY, PCMM10) at a sampling rate of $44.1 \mathrm{kHz}$ with 16-bit quantization. The environmental noise levels were monitored using a sound level meter (Conrad Electronic, Voltcraft Datalogger 322) and maintained at $<50 \mathrm{~dB}$ SPL. The microphone was positioned $15 \mathrm{~cm}$ from the lips and remained at that distance throughout the recording session. Speakers were asked to produce the vowel /a/ three times as long and as steadily as possible. Speakers were also encouraged to keep their pitch and loudness levels constant. The examiner then gave a complete model of the task. Breaks were provided between trials until the speaker felt comfortable and ready for the next trial. A total of 126 trials (42 speakers $\times 3$ repetitions per speaker) were recorded.

\section{Perceptual Estimation of Breathiness}

For perceptual ratings, 3-second vowel samples taken from the middle of the second repetition from each speaker were used. Perceptual judgment was first obtained on the GRBAS scale by two speech language pathologists (S.J.L. and D.L.), who both had clinical experience of vocal assessment for more than 3 years in a Department of Otolaryngology. Twenty college students majoring in speech language pathology participated as listeners for the DME ratings. These listeners were all Korean native female speakers and reported normal hearing. None of them had extensive clinical experience with voice disorders, although all had completed an undergraduate course in voice disorders.

In our DME method with a modulus of 50 [40, 41], a midranged stimulus was arbitrarily selected by the authors as the standard; higher magnitude estimates meant more perceived breathiness, while lower magnitude estimates meant less perceived breathiness. Prior to the experiment, listeners were provided with a brief training on the DME procedure and various voice qualities with emphasis on breathiness. Our instruction defined breathy voice quality as 'audible air escape in the voice' [42]. The stimuli were presented to all listeners simultaneously using a laptop computer and stereo speakers. All listening experiments took place in a quiet room, and listeners were blinded to the age of the speakers. After the background noise levels were controlled as noted above, the modulus stimulus was played. After that, 42 randomized stimuli were played, and 4 stimuli were replayed for reliability testing. The modulus was replayed for reference after every 5 stimulus presentations. In total, 920 ratings ( 20 listeners $\times 42$ stimuli, plus 80 ratings for reliability testing) were made by the listeners. No breaks or repeated presentations of the samples were allowed. In both listening tasks, the listeners judged all vowel stimuli pooled across the two age groups in a random order. After the procedures, the mean DME score for each participant was calculated for further analysis.

Approximately 10\% (4 of 42) of the total stimuli were randomly chosen and replayed to the listeners to obtain intrajudgement reliability. For the GRBAS ratings, inter- and intrarater reliability was evaluated by the intraclass coefficient (ICC), which yielded values of 0.905 (two-way mixed model) and 0.928 (one-way random model). ICCs for inter- and intrareliability of DME scores were 0.914 and 0.728 , respectively.

\section{Acoustic Analysis}

Acoustic measurements were performed using TF32 [43]. First, the relative amplitudes of the two harmonics $(\mathrm{H} 1, \mathrm{H} 2)$ and two formant amplitudes (A1, A3) of the vowel /a/ were measured from 
Table 1. Perceptual estimates for each age group

\begin{tabular}{lllllll}
\hline Age group & Grade & Roughness & Breathiness & Asthenia & Strain & DME ratings \\
\hline YW & $1.0(0.25)$ & $0.0(1.0)$ & $1.0(0.5)$ & $0.0(0.0)$ & $0.0(0.0)$ & $55.96(13.61)$ \\
EW & $1.5(1.0)$ & $1.0(1.0)$ & $1.0(1.0)$ & $0.0(0.0)$ & $0.0(0.0)$ & $64.45(15.78)$ \\
\hline
\end{tabular}

Median (interquartile range) for the GRBAS scale and mean (standard deviation) for DME ratings are presented.

Table 2. Group means and SDs for $\mathrm{H} 1-\mathrm{H} 2, \mathrm{H} 1-\mathrm{A} 1$, and $\mathrm{H} 1-\mathrm{A} 3$ (dB)

\begin{tabular}{|c|c|c|c|c|c|c|}
\hline \multirow{2}{*}{$\begin{array}{l}\text { Acoustic } \\
\text { parameter }\end{array}$} & \multirow[t]{2}{*}{ Age group } & \multicolumn{5}{|l|}{ Window } \\
\hline & & 1 & 2 & 3 & 4 & 5 \\
\hline \multirow[t]{2}{*}{$\mathrm{H} 1-\mathrm{H} 2$} & YW & $-0.067(3.353)$ & $0.324(3.478)$ & $0.803(3.406)$ & $1.278(2.862)$ & 1.127 (2.997) \\
\hline & EW & $-1.354(3.374)$ & $-1.757(3.313)$ & $-1.622(3.564)$ & $-1.946(3.436)$ & $-1.651(3.797)$ \\
\hline \multirow[t]{2}{*}{ H1-A1 } & YW & $-13.516(6.995)$ & $-12.808(6.669)$ & $-13.100(6.926)$ & $-12.768(6.874)$ & $-13.362(7.149)$ \\
\hline & EW & $-15.441(5.969)$ & $-15.737(6.485)$ & $-16.062(6.662)$ & $-16.578(7.702)$ & $-16.838(7.856)$ \\
\hline \multirow[t]{2}{*}{$\mathrm{H} 1-\mathrm{A} 3$} & YW & $-1.952(7.223)$ & $-1.787(6.321)$ & $-1.897(7.225)$ & $-2.037(7.281)$ & $-1.767(8.010)$ \\
\hline & EW & $-3.729(7.858)$ & $-3.765(8.350)$ & $-3.625(7.458)$ & $-3.278(8.920)$ & $-3.137(8.563)$ \\
\hline
\end{tabular}

Windows 1, 2, 3, 4, and 5 stand for 1-second-long windows centered at the time points $1.5 \mathrm{~s}$ away from the onset, 25, 50, and 75\%, and $1.5 \mathrm{~s}$ away from the offset of the prolonged vowel production, respectively.

1-second-long windows centered around five temporal points throughout the prolonged vowel, as described above. The initial and final $1.5 \mathrm{~s}$ were excluded from the analysis considering their potential position effect $[4,44,45]$. For the fixation of the temporal points of each trial, the duration of the prolonged vowel was measured using TF32, and the exact five temporal points were calculated. For each temporal point, peak amplitudes at the frequencies of $\mathrm{H} 1, \mathrm{H} 2, \mathrm{~A} 1$, and $\mathrm{A} 3$ were measured, and then the three acoustic parameters $\mathrm{H} 1-\mathrm{H} 2, \mathrm{H} 1-\mathrm{A} 1$, and $\mathrm{H} 1-\mathrm{A} 3$ were derived from those measurements. Approximately 10\% (180 out of 1,890) of data was randomly chosen to compute the reliability of acoustic measurements, which yielded ICC values of 0.914 and 0.970 for inter- and intrarater reliability (two-way mixed and one-way random model, respectively.

\section{Results}

The Mann-Whitney $U$ test results indicated that both the YW and EW groups showed no statistically significant difference in vowel duration $(\mathrm{p}=0.069$; YW: median $=$ $12.93 \mathrm{~s}$, interquartile range $=3.81$, and $\mathrm{EW}$ : median $=$ $11.60 \mathrm{~s}$, interquartile range $=3.83$ ). Therefore, the time points of the acoustic measurement were not different between the two groups not only in the proportionate but also absolute values.

Aging Effect on Korean Female Voice

\section{Perceptual Ratings of Breathiness}

For the ' $\mathrm{B}$ ' ratings in GRBAS, the Mann-Whitney $U$ test revealed no significant difference in the mean breathiness ratings between the two groups $(p=0.596$; YW: median $=1.0$, interquartile range $=0.5$, and $\mathrm{EW}$ : median $=1.0$, interquartile range $=1.0)$. Other ratings did not significantly differ either: grade $(\mathrm{p}=0.067)$, roughness $(\mathrm{p}=0.124)$, asthenia $(\mathrm{p}=1.000)$, and strain $(\mathrm{p}=0.901)$. With respect to mean DME scores, a Shapiro-Wilk test for normality showed no significance for each group ( $p=0.094$ for $Y W, p=0.053$ for EW). DME estimates did not show a significant difference between the two groups $(\mathrm{t}=-1.867, \mathrm{p}=0.069$; $\mathrm{YW}:$ mean $=55.96, \mathrm{SD}=13.61$, and $\mathrm{EW}:$ mean $=64.45$, $\mathrm{SD}=15.78)$. Results of perceptual ratings are provided in table 1 .

\section{Effect of Age and Measurement Point on Acoustic \\ Variables}

Descriptive results are summarized in table 2. For each group, means and standard deviations of acoustic measures at each measurement point are provided. All values are presented in decibels. A mixed-model analysis of variance (age group by measurement point) was per- 


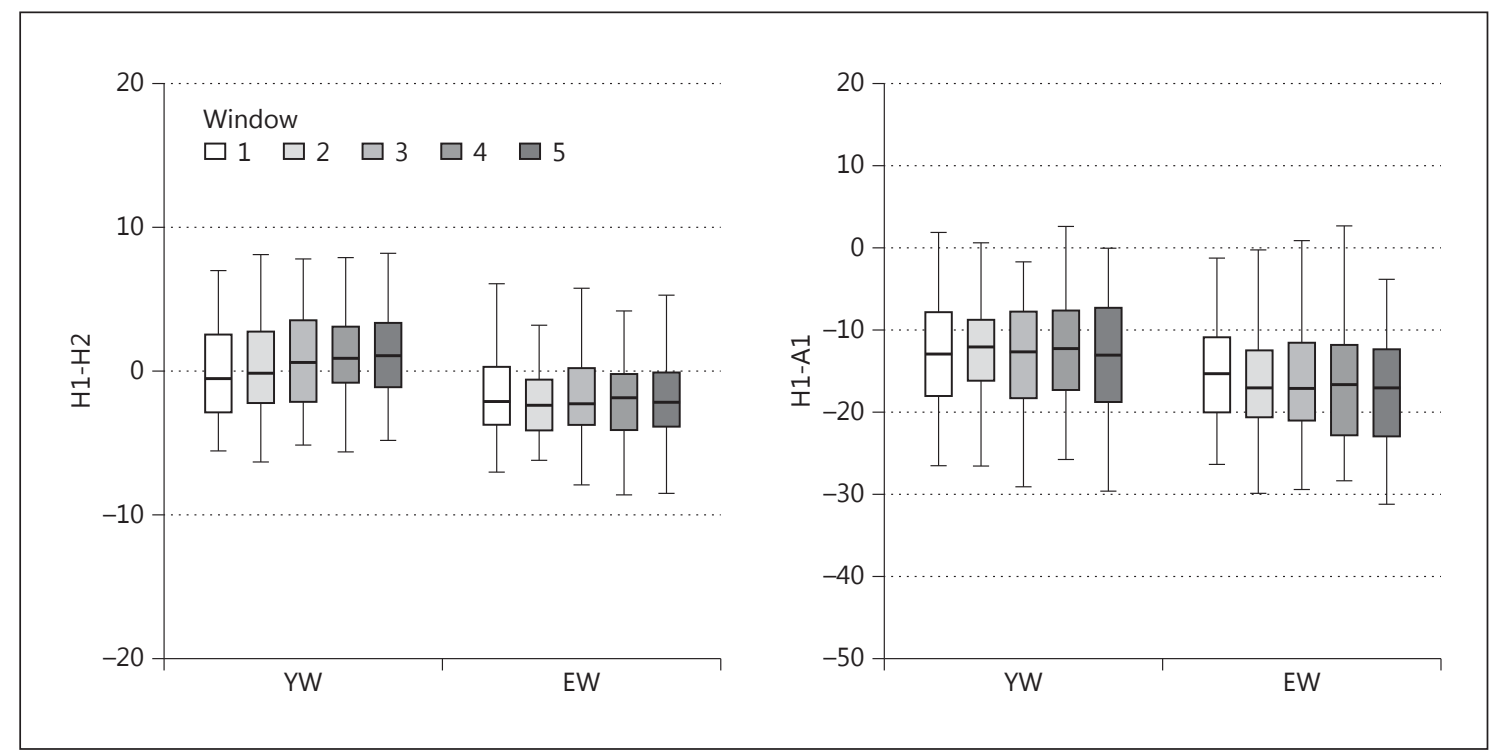

Fig. 1. Group comparison of $\mathrm{H} 1-\mathrm{H} 2, \mathrm{H} 1-\mathrm{A} 1$. The median is indicated by the solid line in the bar. The five time points are labeled as window $1,2,3,4$, and 5, respectively.

formed to investigate the effect of age and measurement point on each acoustic variable. Results indicated a significant main effect for age group. Two variables, $\mathrm{H} 1-\mathrm{H} 2$ $[\mathrm{F}(1,124)=21.482, \mathrm{p}<0.001]$ and $\mathrm{H} 1-\mathrm{A} 1[\mathrm{~F}(1,124)=$ $4.566, \mathrm{p}=0.035]$ of EW were significantly different in EW compared to YW, with more negative values for the EW group. The H1-A3 index did not exhibit a significant difference.

No significant difference in acoustic measures was found between all possible pairs of measurement time points. Figure 1 shows a box and whisker plot of $\mathrm{H} 1-\mathrm{H} 2$ and $\mathrm{H} 1-\mathrm{A} 1$ as a function of age and measurement points.

\section{Acoustic Correlates of Breathiness}

A stepwise regression analysis was conducted to investigate the relationship between the DME values of breathiness and each acoustic parameter. Breathiness was represented by the mean DME scores of each second trial, and acoustic parameters by the measurements at the $50 \%$ point of each second trial. Of the three acoustic parameters, only H1-A1 was significantly regressed against the mean DME scores. Figure 2 shows that H1A1 ( $p=0.001)$ explained $22.9 \%$ of the perceptual breathiness represented by mean DME scores. The direction of the function is in the expected direction: greater $\mathrm{H} 1-\mathrm{A} 1$ values were associated with increasing values of perceived breathiness.

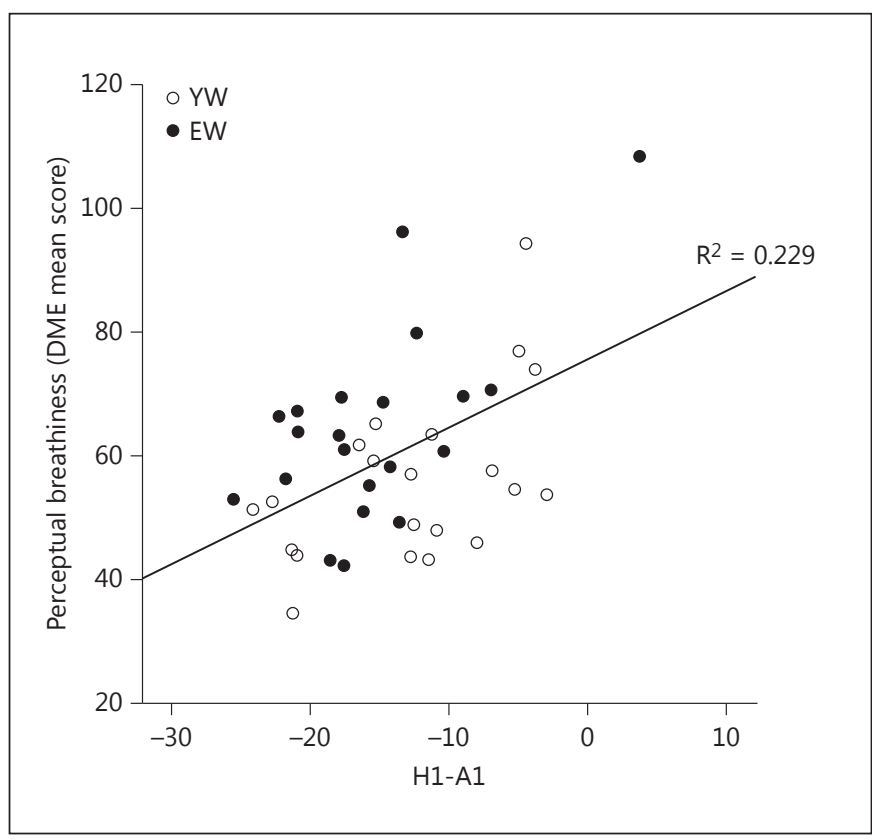

Fig. 2. Perceptual breathiness as a function of H1-A1.

\section{Discussion}

Our findings may be summarized as follows: (1) the vowel duration of the EW group was equivalent to that of the YW group; (2) no difference was perceived by the lis- 
teners in the degree of breathiness between the two age groups, either for the ' $\mathrm{B}$ ' scale of GRBAS or DME scale values; (3) $\mathrm{H} 1-\mathrm{H} 2$ and $\mathrm{H} 1-\mathrm{A} 1$ were significantly greater and more negative for the EW group, and (4) the measure H1-A1 was significantly related to scaled breathiness using DME.

The fact that the two groups did not exhibit differences in vowel duration and perceived breathiness raised a question about the age point when the voice starts exhibiting aging effects, at least on the vowel prolongation task. This doubt was strengthened by the findings that the other GRBAS component ratings also did not differ between the groups. Although menopause begins at about 50 years (approximately 3 years earlier in Korean women) [20], there are other factors that might have affected the detection of perceptual voice changes, such as the use of a different acoustic analysis (e.g., traditional periodicity-based spectral analysis vs. cepstral analysis) and whether or not high vocal demands had been imposed on the female participants [46]. It is also possible that the age groups to which our participants mostly belonged could not fully reflect the effect of aging, at least perceptually, possibly owing to a significantly improved health-related quality of life and increased average life expectancy (for Korean females, 20 years increase over the last 20 years [47]).

Although no difference between the groups was perceptually detected, two acoustic parameters successfully differentiated the groups; in absolute terms, $\mathrm{H} 1-\mathrm{H} 2$ and H1-A1 were significantly greater for EW compared to YW. The greater difference was in the negative direction, indicating less tilt in the spectra of older speakers. This may support the notion that acoustic methods might have better sensitivity to subtle voice-speech characteristics than perceptual methods [48]. This may also reflect that it is relatively difficult to confirm a correlation between the results of acoustic and perceptual assessments compared to other vocal qualities such as hoarseness and roughness [49].

The tendency for the $\mathrm{H} 1-\mathrm{H} 2$ and $\mathrm{H} 1-\mathrm{A} 1$ differences to reflect less tilted spectra in the voices of EW compared to $\mathrm{YW}$ is interesting. As the $\mathrm{H} 1$ amplitude tends to be enhanced for breathy voice compared to modal voice, these results contradict our initial hypothesis that in $\mathrm{H} 1-\mathrm{H} 2$ differences would be greater in EW as compared to YW, reflecting the typical relationship of breathier voices having more positive (more tilted) $\mathrm{H} 1-\mathrm{H} 2$ and $\mathrm{H} 1-\mathrm{A} 1$ values. In a sense, this is not surprising in that the EW were not perceived as breathier. A previous study that showed no difference between age groups in the SPI also supports the current data [23].

Aging Effect on Korean Female Voice
To interpret the current results that $\mathrm{H} 1-\mathrm{H} 2$ and $\mathrm{H} 1-\mathrm{A} 1$ reflected less tilt for EW, the resonance characteristics of Korean vowels should be considered together with aging effects. Prior examinations have reported a substantial difference in formant frequencies between Korean and American English, including /a/; F1, F2, and F3 are located at higher frequencies for Korean /a/ as compared to a language such as English [50]. This difference between languages combined with possible aging influences on harmonic structures (i.e., a decrease in F0 and F1 [51]) might have yielded the current results in Korean EW. In other words, the reduced spectral tilt also hints at the possibility of the influence of the native language or ethnospecific resonance characteristics of vowel /a/ on the acoustic indices of voice quality. In further studies, some corrected measures $[25,52]$ could contribute to the interpretation of our current results, although these measures are not used in the current study, considering the main focus was not on a comparison between ethnic groups.

As for intraspeaker variability across the five measurement points, none of the tested acoustic measures exhibited variations between all possible pairs of measurement points, which implies that any temporal point during vowel prolongation would appropriately represent the characteristics of the vocal quality at least for healthy speakers and for the measures included in this study.

Finally, the findings of the current study indicated that one parameter, H1-A1, was significantly correlated with DME values of breathiness when all speakers were pooled, suggesting its greater sensitivity to the perceptual degree of breathiness than the other two variables. The underlying physiology of this measure, as discussed in previous studies, is that it proportionally reflects the glottal width during vocal fold vibration $[31,35,53,54]$. Another perceptual voice characteristic that could have influenced the current results is 'roughness', as is acoustically correlated with an increase in noise levels throughout the spectrum [52]. In our data, the median of the 'roughness' ratings for EW was 1.0, although they reported no history of pathological voice problems. In addition to their acoustic relevance, the presence of the rough voice quality might have influenced the perceptual DME ratings of breathiness, which in turn possibly affected the correlation between the measurements of acoustic and perceptual breathiness.

This study is not without limitations. First, as previously addressed, the higher age in elderly speakers is likely to provide a clear distinction between the age groups, but may not be sufficiently high to reflect all aging differences. Second, the use of corrected spectral measures [e.g., 52] may yield different results. Furthermore, cross-

Folia Phoniatr Logop 2015;67:300-307 DOI: $10.1159 / 000445290$ 
language experiments where a direct comparison is made between multiple languages to tease out the confounding effect of language and age, or where listeners estimate vocal quality within and across languages would strengthen the preliminary suggestion that the degree of breathy voice and spectral tilt may vary as a function of language. Lastly, a bigger sample size may help to draw a firm conclusion about the characteristics of the Korean female aging voice.

\section{Acknowledgement}

This study was partially supported by a National Institute on Deafness and Other Communication Disorders grant (NIDCD 012405).

\section{Disclosure Statement}

The authors report no conflicts of interest.

\section{References}

1 Linville SE: Vocal Aging. San Diego, Singular Thomson Learning, 2001.

2 Torre P 3rd, Barlow JA: Age-related changes in acoustic characteristics of adult speech. J Commun Disord 2009;42:324-333.

3 Schötz S: Acoustic analysis of adult speaker age; in Müller C (ed): Speaker Classification I. Lecture Notes in Computer Science. Berlin, Springer, 2007, pp 88-107.

4 Stathopoulos ET, Huber JE, Sussman JE: Changes in acoustic characteristics of the voice across the life span: measures from individuals 4-93 years of age. J Speech Lang Hear Res 2011;54:1011-1021.

5 Biever DM, Bless DM: Vibratory characteristics of the vocal folds in young adult and geriatric women. J Voice 1989;3:120-131.

6 Wilcox KA, Horii Y: Age and changes in vocal jitter. J Gerontol 1980;35:194-198.

7 Gorham-Rowan MM, Laures-Gore J: Acoustic-perceptual correlates of voice quality in elderly men and women. J Commun Disord 2006;39:171-184.

8 Goy H, Fernandes DN, Pichora-Fuller MK, van Lieshout P: Normative voice data for younger and older adults. J Voice 2013;27: 545-555.

9 Södersten M, Lindestad PA: Glottal closure and perceived breathiness during phonation in normally speaking subjects. J Speech Hear Res 1990;33:601-611.

10 Schneider B, Bigenzahn W: Influence of glottal closure configuration on vocal efficacy in young normal-speaking women. J Voice 2003; 17:468-480

11 Linville SE: Source characteristics of aged voice assessed from long-term average spectra. J Voice 2002;16:472-479.

12 Hillenbrand J, Houde RA: Acoustic correlates of breathy vocal quality: dysphonic voices and continuous speech. J Speech Hear Res 1996; 39:311-321.

13 Behlau M, Zambon F, Guerrieri AC, Roy N: Epidemiology of voice disorders in teachers and nonteachers in Brazil: prevalence and adverse effects. J Voice 2012;26:665.e669-e618.
14 da Silva PT, Master S, Andreoni S, Pontes P, Ramos LR: Acoustic and long-term average spectrum measures to detect vocal aging in women. J Voice 2011;25:411-419.

15 Radish Kumar B, Bhat JS, Mukhi P: Vowel harmonic amplitude differences in persons with vocal nodules. J Voice 2011;25:559-561.

16 Szakay A: Voice quality as a marker of ethnicity in New Zealand: from acoustics to perception 1. J Socioling 2012;16:382-397.

17 Trent SA: Voice quality: listener identification of African-American versus Caucasian speakers. J Acoust Soc Am 1995;98:29362936.

18 Kreiman J, Gerratt BR: Perceptual sensitivity to first harmonic amplitude in the voice source. J Acoust Soc Am 2010;128:20852089.

19 Esposito CM: The perception of pathologically-disordered phonation by Gujarati, English, and Spanish listeners. Lang Speech 2011;54: 415-430.

20 Hong JS, Yi SW, Kang HC, Jee SH, Kang HG, Bayasgalan G, Ohrr H: Age at menopause and cause-specific mortality in South Korean women: Kangwha cohort study. Maturitas 2007:56:411-419.

21 Byeon H: Relationships among smoking, organic, and functional voice disorders in Korean general population. J Voice 2015;29: 312-316.

22 Jin SM, Kwon KH, Kang HG: Acoustic and stroboscopic characteristics of normal person's voices with advancing age. J Korean Soc Logop Phoniatr 1997;8:44-48.

23 Kim HT, Cho SH, Youn SM, Sun DI, Kim MS: The changes and characteristics of acoustic parameters with aging in Korean. Korean J Otolaryngol Head Neck Surg 2000;43:69-74.

24 Wang YT, Kent RD, Kent JF, Duffy JR, Thomas JE: Acoustic analysis of voice in dysarthria following stroke. Clin Linguist Phon 2009;23: 335-347.

25 Callan DE, Kent RD, Roy N, Tasko SM: Selforganizing map for the classification of normal and disordered female voices. J Speech Lang Hear Res 1999;42:355-366.
26 Kent RD, Vorperian HK, Duffy JR: Reliability of the multi-dimensional voice program for the analysis of voice samples of subjects with dysarthria. Am J Speech Lang Pathol 1999;8: 129-136.

27 Xue SA, Deliyski D: Effects of aging on selected acoustic voice parameters: preliminary normative data and educational implications. Educ Gerontol 2001;27:159-168.

28 Nishi K, Strange W, Akahane-Yamada R, Kubo R, Trent-Brown SA: Acoustic and perceptual similarity of Japanese and American English vowels. J Acoust Soc Am 2008;124: 576-588.

29 Speyer R, Bogaardt HC, Passos VL, Roodenburg NP, Zumach A, Heijnen MA, Baijens LW, Fleskens SJ, Brunings JW: Maximum phonation time: variability and reliability. J Voice 2010;24:281-284.

30 Wayland R, Jongman A: Acoustic correlates of breathy and clear vowels: the case of Khmer. J Phon 2003;31:181-201.

31 Hanson HM: Glottal characteristics of female speakers: acoustic correlates. J Acoust Soc Am 1997;101:466-481.

32 Shrivastav R: The use of an auditory model in predicting perceptual ratings of breathy voice quality. J Voice 2003;17:502-512.

33 Hanson HM, Chuang ES: Glottal characteristics of male speakers: acoustic correlates and comparison with female data. J Acoust Soc Am 1999;106:1064-1077.

34 Thati SA, Bollepalli B, Bhaskararao P, Yegnanarayana $B$ : Analysis of breathy voice based on excitation characteristics of speech production. 2012 Int Conf IEEE, 2012, pp 1-5.

35 Morris RJ, Gorham-Rowan MM, Harmon $A B$ : The effect of initiating oral contraceptive use on voice: a case study. J Voice 2011;25: 223-229.

36 De Bodt MS, Wuyts FL, Van de Heyning PH, Croux C: Test-retest study of the GRBAS scale: influence of experience and professional background on perceptual rating of voice quality. J Voice 1997;11:74-80. 
37 Nemr K, Simoes-Zenari M, Cordeiro GF, Tsuji D, Ogawa AI, Ubrig MT, Menezes MH: GRBAS and CAPE-V scales: high reliability and consensus when applied at different times. J Voice 2012;26:812.e817-e822.

38 Weismer G, Laures JS: Direct magnitude estimates of speech intelligibility in dysarthria: effects of a chosen standard. J Speech Lang Hear Res 2002;45:421-433.

39 Whitehill TL, Lee AS, Chun JC: Direct magnitude estimation and interval scaling of hypernasality. J Speech Lang Hear Res 2002;45: $80-88$.

40 Gescheider GA: Psychophysics: Method and Theory. Hillsdale, Lawrence Erlbaum, 1976.

41 Schiavetti N: Scaling procedures for the measurement of speech intelligibility; in Kent RD (ed): Intelligibility in Speech Disorders. Amsterdam, John Benjamins Publishing Company, 1992, pp 11-34.

42 Kempster GB, Gerratt BR, Verdolini Abbott $\mathrm{K}$, Barkmeier-Kraemer J, Hillman RE: Con- sensus auditory-perceptual evaluation of voice: development of a standardized clinical protocol. Am J Speech Lang Pathol 2009;18: 124-132.

43 Milenkovic P: TF32. Madison, University of Wisconsin-Madison, 2005

44 Awan SN: The aging female voice: acoustic and respiratory data. Clin Linguist Phon 2006;20:171-180.

45 Sapienza CM, Stathopoulos ET: Speech task effects on acoustic and aerodynamic measures of women with vocal nodules. J Voice 1995;9:413-418.

46 Friedman AD: The impact of menopause on voice: past, present, and future. Menopause 2011;18:248-250

47 Korean Statistical Information Service (KOSIS): Life expectancy at birth. 2011. http://kosis.kr/.

48 Duffy JR: Motor speech disorders: substrates, differential diagnosis, and management. Amsterdam, Elsevier Health Sciences, 2013.
49 Eskenazi L, Childers DG, Hicks DM: Acoustic correlates of vocal quality. J Speech Lang Hear Res 1990;33:298-306.

50 Yang B: A comparative study of American English and Korean vowels produced by male and female speakers. J Phon 1996;24:245-261.

51 Reubold U, Harrington J, Kleber F: Vocal aging effects on F0 and the first formant: a longitudinal analysis in adult speakers. Speech Commun 2010;52:638-651.

52 Iseli M, Shue YL, Alwan A: Age, sex, and vowel dependencies of acoustic measures related to the voice source. J Acoust Soc Am 2007; 121:2283-2295.

53 de Krom G: Some spectral correlates of pathological breathy and rough voice quality for different types of vowel fragments. J Speech Hear Res 1995;38:794-811.

54 Klatt DH, Klatt LC: Analysis, synthesis, and perception of voice quality variations among female and male talkers. J Acoust Soc Am 1990;87:820-857. 